\title{
The Identification of Convex Function on Riemannian Manifold
}

\author{
Li Zou, ${ }^{1,2}$ Xin Wen, ${ }^{1}$ Hamid Reza Karimi, ${ }^{3}$ and Yan Shi ${ }^{4}$ \\ ${ }^{1}$ School of Computer and Information Technology, Liaoning Normal University, Dalian 116081, China \\ ${ }^{2}$ State Key Laboratory for Novel Software Technology, Nanjing University, Nanjing 210093, China \\ ${ }^{3}$ Department of Engineering, Faculty of Engineering and Science, University of Agder, Grimstad, Norway \\ ${ }^{4}$ General Education Center (Kumamoto/Aso), Tokai University, Kumamoto, Japan
}

Correspondence should be addressed to Hamid Reza Karimi; hamid.r.karimi@uia.no

Received 30 December 2013; Accepted 26 February 2014; Published 7 April 2014

Academic Editor: Yuxin Zhao

Copyright $\odot 2014$ Li Zou et al. This is an open access article distributed under the Creative Commons Attribution License, which permits unrestricted use, distribution, and reproduction in any medium, provided the original work is properly cited.

\begin{abstract}
The necessary and sufficient condition of convex function is significant in nonlinear convex programming. This paper presents the identification of convex function on Riemannian manifold by use of Penot generalized directional derivative and the Clarke generalized gradient. This paper also presents a method for judging whether a point is the global minimum point in the inequality constraints. Our objective here is to extend the content and proof the necessary and sufficient condition of convex function to Riemannian manifolds.
\end{abstract}

\section{Introduction}

Manifold is the space with the local property of Euclidean space. We often judge the warp of the space by measuring its space. The standard of the measurement is called metric. Metric is an intrinsic property and the space with metric is called Riemannian space. Riemann manifold is a differential manifold which has Riemannian metric. Manifold learning attempts to obtain the intrinsic structure of non-linearly distributed data, which can be used in non-linear dimensionality reduction (NLDR). We can find the meaningful low dimensional structure hidden in the high-dimensional observation data by the non-linear dimension reduction of high data space [1]. Expanding the optimization based on linear space to the nonlinear space (such as Riemannian manifold) is a hot topic in this research field [2]. Convex function is a kind of special function that has a broad application, especially has a wide range of applications in the areas of optimization and mathematical programming, cybernetics, and other fields. Since the useful data is usually not a linear combination of the features in the real world that the nonlinear convex programming problem has become a new research subject [3-5]. In order to have a more indepth study of nonsmooth and its related problems, several important tools for analyzing nonsmooth problem have been extended from European space to Riemannian Manifold.
In 2004, Ledyacv and Zhu established the concept and algorithm of subdifferential of nonsmooth function in Riemannian manifold and proved that the solution of HamiltonJacobi equation defined on Riemannian manifold is unique. They also discussed the concept and variational principle of approximate subdifferential in Riemannian manifold $[6,7]$. In 2005, Azagra and others discussed the J. Ferrera subdifferential which was defined on finite dimensional Riemannian manifold and relevant limit subdifferential and analyzed the issues of differential inclusion which were defined on differential manifold by using the concept of generalized derivative $[8,9]$. Liu and others expanded (MP) problem from linear space to differential manifold and Fritz John necessary optimality conditions on Riemann manifold was given [10]. In 2008, Gang and Sanyang solved the nondifferentiable multiobjective optimization problems with equality and inequality constraints from Euclidean space to Riemannian manifolds and derived the Fritz-John necessary conditions with generalized gradient formula for weak Pareto optimal solutions from Ekeland variational principle [11]. Wang et al. established the semilocal convergence of sequences which was generated by the Gauss-Newton method (with quasiregular initial points) for the convex composite function $h$ 。 $F$ on Riemannian manifold [12] by using the majorizing function technique. Bento et al. presented a steepest descent method with Armijo's rule for multicriteria optimization in 
the Riemannian context. With assuming quasiconvexity of the multicriteria function and nonnegative curvature of the Riemannian manifold, they proved full convergence of the sequence to a critical Pareto point [13].

Convex programming is of great significance in the study of nonlinear programming theory. Based on the above research results, we will parallel extend the identification of convex function and the optimality conditions of constraint problems from linear space to Riemannian manifold in order to solve the convex programming problem in Riemannian manifold. This paper is set out as follows. In Section 2, we briefly review some preliminary concepts. In Section 3, we introduce the identification of convex function on Riemannian manifold. We make some concluding remarks and suggest future research in this area in Section 4.

\section{Preliminaries}

Let $R^{n}$ be an $n$-dimensional Euclidean space; the $i$ th coordinate of point $p \in R^{n}$ is denoted by $(p)^{i}$; that is ()$^{i}$ is the $i$ th coordinate function of $R^{n}$.

Definition 1 (see [14]). Let $M$ be a Hausdorff topological space. If every point $p$ of $M$ has an open neighborhood $U \subset M$, such that an open subset of $U$ and $n$-dimensional Euclidean space is homeomorphous, then $M$ is called an $n$ dimensional topological manifold.

Definition 2 (see [10]). Let $f(x)$ be a real-valued function defined on Riemannian manifold, $x_{0} \in U \subset M$, and let $(U, \varphi)$ be a coordinate plot which contains $x_{0}$. If for all $x, y \in U$, there exists a constant $L$ such that

$$
|f(y)-f(x)| \leq L|\varphi(y)-\varphi(x)| .
$$

Then, $f(x)$ is a function which meets the local Lipschitz condition in the neighborhood of $x_{0}$.

Definition 3 (see [10]). Let function $f: M \rightarrow R$ be a meet local Lipschitz condition in the neighborhood of the point $x \in M$ and $(U, \varphi)$ be a coordinate plot which contains $x$. The Clarke generalized directional derivative of $f$ at $x$ along the direction of $v \in T_{x} M$ is denoted by

$$
\begin{aligned}
& f^{\circ}(x ; v) \\
& \quad=\lim _{y \rightarrow \varphi(x)} \sup _{t \downarrow 0} \frac{f \circ \varphi^{-1}\left(y+t \varphi_{*_{x}}(v)\right)-f \circ \varphi^{-1}(y)}{t} \\
& \quad=\left(f \circ \varphi^{-1}\right)^{\circ}\left(\varphi(x) ; \varphi_{*_{x}}(v)\right),
\end{aligned}
$$

where $t \rightarrow 0$.

Definition 4 (see [10]). Let $f(x)$ be a function denoted in Riemannian manifold $(M, g)$ and meet local Lipschitz condition in the neighborhood of $x$. Then, the element of the subset of cotangent space $T_{x}^{*} M$ of $x$

$$
\partial f(x)=\left\{\xi \in T_{x}{ }^{*} M \mid f^{\circ}(x ; v) \geq(\xi, v), \forall v \in T_{x} M\right\}
$$

is said to be Clarke generalized gradient in which $f$ is at $x$.
The norm of generalized gradient is denoted by

$$
\|\xi\|_{\varphi}:=\sup \left\{(\xi, v), v \in T_{x} M,\|v\| \leq 1\right\} .
$$

Definition 5 (see [15]). Let $f: M \rightarrow R$ meet local Lipschitz condition in the neighborhood of $x \in M$ and let $(U, \varphi)$ be a coordinate plot which contains $x$. We define Penot generalized directional derivative of $f$ at $x$ along the direction of $v \in T_{x} M$ as follows:

$$
\begin{aligned}
\underline{d} f & (x ; v) \\
& =\liminf _{u \rightarrow v t \downarrow 0} \frac{f \circ \varphi^{-1}\left(y+t \varphi_{*_{x}}(u)\right)-f \circ \varphi^{-1}(y)}{t} \\
& =\underline{d}\left(f \circ \varphi^{-1}\right)\left(\varphi(x) ; \varphi_{*_{x}}(v)\right), \\
\bar{d} f & (x ; v) \quad-\limsup \frac{f \circ \varphi^{-1}\left(y+t \varphi_{*_{x}}(u)\right)-f \circ \varphi^{-1}(y)}{t} \\
& =\bar{d}\left(f \circ \varphi^{-1}\right)\left(\varphi(x) ; \varphi_{*_{x}}(v)\right), \\
\underline{d}^{2} f & (x ; v ; w) \\
& =\liminf _{u \rightarrow \omega}\left(f \circ \varphi^{-1}\left(y+t \varphi_{*_{x}}(v)+t^{2} \varphi_{*_{x}}(u)\right)\right. \\
& =\lim _{u \rightarrow \omega} \sup _{t \downarrow 0}\left(f \circ \varphi^{-1}\left(y+t \varphi_{*_{x}}(v)+t^{2} \varphi_{*_{x}}(u)\right)\right. \\
\bar{d}^{2} f(x ; v ; w) & \left.-f \circ \varphi^{-1}(y)-t \bar{d} f(x ; v)\right)\left(t^{-2}\right) .
\end{aligned}
$$

If $\underline{d} f(x ; v)=\bar{d} f(x ; v)$, we define $d f(x ; v)$ as Penot generalized directional derivative of $f$ at $x$ along the direction of $v \in T_{x} M$.

If $\underline{d}^{2} f(x ; v ; \omega)=\bar{d}^{2} f(x ; v ; \omega)$, we define $d^{2} f(x ; v ; \omega)$ to represent second-order Penot generalized directional derivative of $f$ at $x$ along the direction of $v \in T_{x} M$.

If both $d f(x ; v)$ and $d^{2} f(x ; v ; \omega)$ exist, then

$$
\begin{array}{r}
d^{2} f(x ; v ; \omega) \\
=\lim _{y \rightarrow \varphi(x), t \downarrow 0}\left(f \circ \varphi^{-1}\left(y+t \varphi_{*_{x}}(v)+t^{2} \varphi_{*_{x}}(\omega)\right)\right. \\
\left.\quad-f \circ \varphi^{-1}(y)-t d f(x ; v)\right)\left(t^{-2}\right) .
\end{array}
$$

\section{The Identification of Convex Function on Riemannian Manifold}

Theorem 6 (first-order necessary and sufficient condition of convex function). Let $M$ be an m-dimensional Riemannian manifold and let $U$ be a geodesic convex set, where $U \subset M$. If $f$ : $U \rightarrow R$ meets local Lipschitz condition in $U$, then the necessary 
and sufficient condition that $f$ is geodesic convex function in $U$ is as follows. For $\forall x, y \in U$, there exists

$$
\begin{aligned}
f & \circ \varphi^{-1}\left(y+\varphi_{*_{x}}(v)\right) \\
& \geq f \circ \varphi^{-1}(y)+\nabla\left(f \circ \varphi^{-1}\right)(y)^{T}(y-x) .
\end{aligned}
$$

Proof (necessary condition). For $\forall t \in[0,1]$, there exists

$$
\begin{aligned}
& \varphi^{-1}(y)+t\left[\varphi^{-1}\left(y+\varphi_{*_{x}}(v)\right)-\varphi^{-1}(y)\right] \\
& \quad=t \varphi^{-1}\left(y+\varphi_{*_{x}}(v)\right)+(1-t) \varphi^{-1}(y) \in U .
\end{aligned}
$$

Then, according to the first-order Taylor expansion,

$$
\begin{aligned}
f & \circ\left(\varphi^{-1}(y)+t\left[\varphi^{-1}\left(y+\varphi_{*_{x}}(v)\right)-\varphi^{-1}(y)\right]\right) \\
& =f \circ \varphi^{-1}(y)+t \nabla\left(f \circ \varphi^{-1}\right)(y)^{T}(y-x)+o(t) .
\end{aligned}
$$

Since $f$ is geodesic convex function in $U$, then

$$
\begin{aligned}
f & \circ\left(\varphi^{-1}(y)+t\left[\varphi^{-1}\left(y+\varphi_{*_{x}}(v)\right)-\varphi^{-1}(y)\right]\right) \\
& =f \circ\left(t \varphi^{-1}\left(y+\varphi_{*_{x}}(v)\right)+(1-t) \varphi^{-1}(y)\right) \\
& \leq t f \circ \varphi^{-1}\left(y+\varphi_{*_{x}}(v)\right)+(1-t) f \circ \varphi^{-1}(y) .
\end{aligned}
$$

Then, from two simultaneous equations of (9) and (10), we get

$$
\begin{aligned}
f & \circ \varphi^{-1}\left(y+\varphi_{*_{x}}(v)\right)+(1-t) f \circ \varphi^{-1}(y) \\
& \geq f \circ \varphi^{-1}(y)+t \nabla\left(f \circ \varphi^{-1}\right)(y)^{T}(y-x)+o(t) ;
\end{aligned}
$$

that is

$$
\begin{aligned}
f & \circ \varphi^{-1}\left(y+\varphi_{*_{x}}(v)\right) \\
& \geq f \circ \varphi^{-1}(y)+\nabla\left(f \circ \varphi^{-1}\right)(y)^{T}(y-x)+\frac{o(t)}{t} .
\end{aligned}
$$

It follows from $t \rightarrow 0^{+}$that $(o(t) / t) \rightarrow 0$; hence,

$$
\begin{aligned}
f & \circ \varphi^{-1}\left(y+\varphi_{*_{x}}(v)\right) \\
& \geq f \circ \varphi^{-1}(y)+\nabla\left(f \circ \varphi^{-1}\right)(y)^{T}(y-x) .
\end{aligned}
$$

Sufficient Condition. For $\forall t \in[0,1]$, let $z=t x+(1-t) y$.

From the known conditions it follows that

$$
\begin{aligned}
& f \circ \varphi^{-1}(y) \\
& \geq f \circ \varphi^{-1}\left(y+(1-t) \varphi_{*_{x}}(v)\right) \\
&+\nabla\left(f \circ \varphi^{-1}\right)\left(y+(1-t) \varphi_{*_{x}}(v)\right)^{T}(x-z), \\
& f \circ \varphi^{-1}\left(y+\varphi_{*_{x}}(v)\right) \\
& \geq f \circ \varphi^{-1}\left(y+(1-t) \varphi_{*_{x}}(v)\right) \\
& \quad+\nabla\left(f \circ \varphi^{-1}\right)\left(y+(1-t) \varphi_{*_{x}}(v)\right)^{T}(y-z) .
\end{aligned}
$$

Hence,

$$
\begin{aligned}
& t f \circ \varphi^{-1}(y) \\
& \geq t f \circ \varphi^{-1}\left(y+(1-t) \varphi_{*_{x}}(v)\right) \\
& \quad+t \nabla\left(f \circ \varphi^{-1}\right)\left(y+(1-t) \varphi_{*_{x}}(v)\right)^{T}(x-z), \\
& (1-t) f \circ \varphi^{-1}\left(y+\varphi_{*_{x}}(v)\right) \\
& \geq(1-t) f \circ \varphi^{-1}\left(y+(1-t) \varphi_{*_{x}}(v)\right) \\
& \quad+(1-t) \nabla\left(f \circ \varphi^{-1}\right)\left(y+(1-t) \varphi_{*_{x}}(v)\right)^{T}(y-z) .
\end{aligned}
$$

Then, from (15) it follows that

$$
\begin{aligned}
t f \circ \varphi^{-1}(y)+(1-t) f \circ \varphi^{-1}\left(y+\varphi_{*_{x}}(v)\right) \\
\geq f \circ \varphi^{-1}\left(y+(1-t) \varphi_{*_{x}}(v)\right) \\
\quad+\nabla\left(f \circ \varphi^{-1}\right)\left(y+(1-t) \varphi_{*_{x}}(v)\right)^{T}(t x+(1-t) y-z) .
\end{aligned}
$$

Since $z=t x+(1-t) y$, then

$$
\begin{aligned}
t f & \circ \varphi^{-1}(y)+(1-t) f \circ \varphi^{-1}\left(y+\varphi_{*_{x}}(v)\right) \\
& \geq f \circ \varphi^{-1}\left(y+(1-t) \varphi_{*_{x}}(v)\right) \\
& =f \circ\left(t \varphi^{-1}(y)+(1-t) \varphi^{-1}\left(y+\varphi_{*_{x}}(v)\right)\right) .
\end{aligned}
$$

Thus, $f$ is geodesic convex function in $U$.

Theorem 7 (first-order necessary and sufficient condition of strictly convex function). Let $M$ be an m-dimensional Riemannian manifold and $U$ be a geodesic convex set. Let $U \subset$ $M, f: U \rightarrow R$ meet local Lipschitz condition in $U$, and then the necessary and sufficient condition that $f$ is geodesic strictly convex function in $U$ is as follows. For $\forall x, y \in U$, there exists

$$
\begin{aligned}
f & \circ \varphi^{-1}\left(y+\varphi_{*_{x}}(v)\right) \\
& >f \circ \varphi^{-1}(y)+\nabla\left(f \circ \varphi^{-1}\right)(y)^{T}(y-x) .
\end{aligned}
$$

Proof (necessary condition). In a similar way with proof of Theorem 6, we get

$$
\begin{aligned}
& f \circ \varphi^{-1}\left(y+\varphi_{*_{x}}(v)\right) \\
& \quad>f \circ \varphi^{-1}(y)+\nabla\left(f \circ \varphi^{-1}\right)(y)^{T}(y-x) .
\end{aligned}
$$

Sufficient Condition. Since $f$ is a strictly convex function, then $f$ is a convex function. For any two different points $x, y$ in which $x, y \in U$, there exists $z=(1 / 2) x+(1 / 2) y$, and then $z \in U$. 
From Theorem 6 it follows that

$$
\begin{aligned}
f \circ & \varphi^{-1}\left(y+\frac{1}{2} \varphi_{*_{x}}(v)\right) \\
& \geq f \circ \varphi^{-1}(y)+\nabla\left(f \circ \varphi^{-1}\right)(y)^{T}(z-x) \\
& =f \circ \varphi^{-1}(y)+\nabla\left(f \circ \varphi^{-1}\right)(y)^{T}\left(\frac{1}{2} x+\frac{1}{2} y-x\right) \\
& =f \circ \varphi^{-1}(y)+\frac{1}{2} \nabla\left(f \circ \varphi^{-1}\right)(y)^{T}(y-x) .
\end{aligned}
$$

Since $f$ is a strictly convex function, then

$$
\begin{aligned}
f \circ & \varphi^{-1}\left(y+\frac{1}{2} \varphi_{*_{x}}(v)\right) \\
& =f \circ\left(\frac{1}{2} \varphi^{-1}(y)+\frac{1}{2} \varphi^{-1}\left(y+\varphi_{*_{x}}(v)\right)\right) \\
& <\frac{1}{2} f \circ \varphi^{-1}(y)+\frac{1}{2} f \circ \varphi^{-1}\left(y+\varphi_{*_{x}}(v)\right) .
\end{aligned}
$$

Then, it follows from (20) and (21) that

$$
\begin{aligned}
\frac{1}{2} f & \circ \varphi^{-1}(y)+\frac{1}{2} f \circ \varphi^{-1}\left(y+\varphi_{*_{x}}(v)\right) \\
& >f \circ \varphi^{-1}(y)+\frac{1}{2} \nabla\left(f \circ \varphi^{-1}\right)(y)^{T}(y-x) ;
\end{aligned}
$$

that is,

$$
\begin{aligned}
f \circ \varphi^{-1}\left(y+\varphi_{*_{x}}(v)\right) \\
\quad>f \circ \varphi^{-1}(y)+\nabla\left(f \circ \varphi^{-1}\right)(y)^{T}(y-x) .
\end{aligned}
$$

Theorem 8 (second-order necessary and sufficient condition of convex function). Let $M$ be an $m$-dimensional Riemannian manifold and $U$ be a geodesic convex set. Let $U \subset M, f$ : $U \rightarrow R$ meet local Lipschitz condition in $U$ and let $(U, \varphi)$ be a coordinate plot which contains $x$, and second-order Penot generalized directional derivative of $f$ at $x$ along the direction of $v \in T_{x} M$ exists. Then, the necessary and sufficient condition that $f$ is geodesic convex function in $U$ is as follows. For any $x \in U$, there exists $d^{2} f(x ; v, w) \geq 0$.

Proof (necessary condition). Assume that for any $x \in U$, there exists $d^{2} f(x ; v, w) \geq 0$. For any two different points $x, y$, from Taylor expansion, it follows that

$$
\begin{aligned}
f \circ \varphi^{-1}\left(y+\varphi_{*_{x}}(v)\right) \\
=f \circ \varphi^{-1}(y)+\nabla\left(f \circ \varphi^{-1}\right)(y)^{T}(y-x) \\
\quad+\frac{1}{2}(y-x)^{T} \nabla^{2}\left(f \circ \varphi^{-1}\right)(z)(y-x) .
\end{aligned}
$$

Note that $z=t x+(1-t) y, 0 \leq t \leq 1$.

Since $U$ is a geodesic convex set, then $z \in U$. From the known conditions it follows that

$$
d^{2} f(x ; v, w) \geq 0 .
$$

Hence,

$$
(y-x)^{T} \nabla^{2}\left(f \circ \varphi^{-1}\right)(z)(y-x) \geq 0
$$

so that

$$
\begin{aligned}
f & \circ \varphi^{-1}\left(y+\varphi_{*_{x}}(v)\right) \\
& \geq f \circ \varphi^{-1}(y)+\nabla\left(f \circ \varphi^{-1}\right)(y)^{T}(y-x) .
\end{aligned}
$$

Therefore, $f$ is geodesic convex function in $U$ according to Theorem 7 .

Sufficient Condition. Since $U$ is a geodesic convex set, thus for any $x \in U$ and any given nonzero vector $l$, there exists a sufficiently small positive number $t$ and thus $x+t l \in U$; it follows from Taylor expansion that

$$
\begin{aligned}
f \circ \varphi^{-1}(x+t l)= & f \circ \varphi^{-1}(x)+t \nabla\left(f \circ \varphi^{-1}\right)(x)^{T} l \\
& +\frac{t^{2}}{2} l^{T} \nabla^{2}\left(f \circ \varphi^{-1}\right)(\bar{x}) l+o\left(t^{2}\right) .
\end{aligned}
$$

Since $f$ is geodesic convex function in $U$, using Theorem 7, we have

$$
f \circ \varphi^{-1}(x+t l) \geq f \circ \varphi^{-1}(x)+t \nabla\left(f \circ \varphi^{-1}\right)(x)^{T} l .
$$

Hence,

$$
\frac{t^{2}}{2} l^{T} \nabla^{2}\left(f \circ \varphi^{-1}\right)(\bar{x}) l+o\left(t^{2}\right) \geq 0 .
$$

Let $t \rightarrow 0^{+}$; then,

$$
\frac{o\left(t^{2}\right)}{t^{2}} \longrightarrow 0
$$

Thus,

$$
l^{T} \nabla^{2}\left(f \circ \varphi^{-1}\right)(\bar{x}) l \geq 0 .
$$

That is, for any $x \in U$, there exists $d^{2} f(x ; v, w) \geq 0$.

Theorem 9 (second-order necessary and sufficient condition of strictly convex function). Let $M$ be an $m$-dimensional Riemannian manifold and $U$ be a geodesic convex set. Let $U \subset$ $M, f: U \rightarrow R$ meet local Lipschitz condition in $U$ and let $(U, \varphi)$ be a coordinate plot which contains $x$ and second-order Penot generalized directional derivative of $f$ at $x$ along the direction of $v \in T_{x} M$ exists. Then, the necessary and sufficient condition that $f$ is geodesic strictly convex function in $U$ is as follows. For any $x \in U$, there exists $d^{2} f(x ; v, w)>0$.

Proof. It is similar to the proof of Theorem 8.

Definition 10. For the mathematical programming problem

$$
\begin{array}{ll}
\min & f(x) \\
\text { st. } & g_{i}(x) \leq 0, \quad i \in I
\end{array}
$$


where $f: M \rightarrow R, g: M \rightarrow R^{l}$ are geodesic convex functions in $U, U$ is an open subset of $M,(U, \varphi)$ is a coordinate plot which contains $x$, and the feasible region of the problem is denoted by

$$
D:=\left\{x \in M \mid g_{i}(x) \leq 0, i \in I\right\} .
$$

Theorem 11. Let $M$ be an m-dimensional Riemannian manifold and let $x^{*}$ be the optimal solution of the problem. If $f$ : $M \rightarrow R, g_{i}: M \rightarrow R, i \in I$ meet local Lipschitz condition in the neighborhood of $x^{*} \in M$ and $g_{i}$ is continuous in $x^{*}$, where $i \notin I$, or the vector group $\nabla\left(g_{i} \circ \varphi^{-1}\right)\left(\varphi\left(x^{*}\right)\right)$ is linearly independent, where $i \in I$, then there exists constant $u_{i}(i \in I)$, such that

$$
\begin{array}{r}
\nabla\left(f \circ \varphi^{-1}\right)\left(\varphi\left(x^{*}\right)\right)+\sum_{i \in I} u_{i} \nabla\left(g_{i} \circ \varphi^{-1}\right)\left(\varphi\left(x^{*}\right)\right)=0, \\
u_{i} \geq 0, \quad i \in I .
\end{array}
$$

In particular, if $g_{i}$ meets local Lipschitz condition in the neighborhood of $x^{*} \in M$, where $i \notin I$, then

$$
\begin{gathered}
\nabla\left(f \circ \varphi^{-1}\right)\left(\varphi\left(x^{*}\right)\right)+\sum_{i=1}^{m} u_{i} \nabla\left(g_{i} \circ \varphi^{-1}\right)\left(\varphi\left(x^{*}\right)\right)=0, \\
u_{i}\left(g_{i} \circ \varphi^{-1}\right)\left(\varphi\left(x^{*}\right)\right)=0, \quad i=1,2, \ldots, m, \\
u_{i} \geq 0, \quad i=1,2, \ldots, m .
\end{gathered}
$$

Theorem 11 is called Kuhn-Tucker theorem or K-T theorem for short. The necessary condition of the minimum point is called K-T condition and the point which meets the K-T condition is called $\mathrm{K}-\mathrm{T}$ point.

Theorem 12 (sufficient condition of the minimum point). Let the minimization problem (33) be a convex programming problem and let $x^{*}$ be a K-T point. Then, $x^{*}$ is the global minimum point.

Proof. For $\forall x$, there exists

$$
g_{i} \circ \varphi^{-1}(\varphi(x)) \leq 0 .
$$

Since $g_{i} \circ \varphi^{-1}\left(\varphi\left(x^{*}\right)\right)$ is geodesic convex function, then

$$
\begin{array}{r}
g_{i} \circ \varphi^{-1}(\varphi(x)) \geq g_{i} \circ \varphi^{-1}\left(\varphi\left(x^{*}\right)\right) \\
+\nabla\left(g_{i} \circ \varphi^{-1}\right)\left(\varphi\left(x^{*}\right)\right)^{T}\left(x-x^{*}\right), \\
\quad i=1,2, \ldots, m .
\end{array}
$$

For $i \in I$, there exists

$$
g_{i} \circ \varphi^{-1}\left(\varphi\left(x^{*}\right)\right)=0 .
$$

Then,

$$
\nabla\left(g_{i} \circ \varphi^{-1}\right)\left(\varphi\left(x^{*}\right)\right)^{T}\left(x-x^{*}\right) \leq 0 .
$$

Since $x^{*}$ is a K-T point, then

$$
\begin{aligned}
& \nabla\left(f \circ \varphi^{-1}\right)\left(\varphi\left(x^{*}\right)\right)=-\sum_{i \in I} u_{i} \nabla\left(g_{i} \circ \varphi^{-1}\right)\left(\varphi\left(x^{*}\right)\right), \\
& u_{i} \geq 0, \quad i \in I, \\
& \nabla\left(f \circ \varphi^{-1}\right)\left(\varphi\left(x^{*}\right)^{T}\right)\left(x-x^{*}\right) \\
& =-\sum_{i \in I} u_{i} \nabla\left(g_{i} \circ \varphi^{-1}\right)\left(\varphi\left(x^{*}\right)\right)^{T}\left(x-x^{*}\right) \geq 0 .
\end{aligned}
$$

Since $f \circ \varphi^{-1}(\varphi(x))$ is geodesic convex function, then

$$
\begin{aligned}
f \circ \varphi^{-1}(\varphi(x)) \geq & f \circ \varphi^{-1}\left(\varphi\left(x^{*}\right)\right) \\
& +\nabla\left(f \circ \varphi^{-1}\right)\left(\varphi\left(x^{*}\right)^{T}\right)\left(x-x^{*}\right), \\
f \circ \varphi^{-1}(\varphi(x)) \geq & f \circ \varphi^{-1}\left(\varphi\left(x^{*}\right)\right) .
\end{aligned}
$$

Thus, $x^{*}$ is the global minimum point.

\section{Conclusions}

In this paper, we introduce the classical Clarke generalized directional derivative and generalized gradient. Then, we extend the identification of convex function and prove the theorem of the first-order necessary and sufficient condition of convex function and strictly convex function to Riemannian manifolds by use of Penot generalized directional derivative. We have also given the second-order necessary and sufficient condition of convex function and strictly convex function and the Kuhn-Tucker theorem and sufficient condition of the minimum point of the inequality constrained optimization problems. In this way, we can do more research about optimization method on differential manifold. In the future, the theorem and the application of the equality constrained optimization problems and the inequality constrained optimization problems will be explored.

\section{Conflict of Interests}

The authors declare that there is no conflict of interests regarding the publication of this paper.

\section{Acknowledgments}

This work is partly supported by the National Natural Science Foundation of China (Grant nos. 61105059, 61175055, 61372187, and 61173100), International Cooperation and Exchange of the National Natural Science Foundation of China (Grant no. 61210306079), Sichuan Key Technology Research and Development Program (Grant no. 2011FZ0051), Radio Administration Bureau of MIIT of China (Grant no. [2011]146), China Institution of Communications (Grant no. [2011]051), and Sichuan Key Laboratory of Intelligent Network Information Processing (Grant no. SGXZD1002-10). 


\section{References}

[1] E. Z. Gao and S. T. Wang, "Minimum squared mean distance based on dimension reduction of riemannian manifold," Computer Engineering and Applications, vol. 49, no. 2, pp. 198-202, 2013.

[2] D. Azagra, J. Ferrera, and F. López-Mesas, "Nonsmooth analysis and Hamilton-Jacobi equations on Riemannian manifolds," Journal of Functional Analysis, vol. 220, no. 2, pp. 304-361, 2005.

[3] S. F. Li, Application of Lipschitz Condition in Convex Function, Chongqing University of Technology, 2011.

[4] Y. Y. Yan, Y. Shi, and F. Liu, "Constrained model predictive control on convex polyhedron stochastic linear parameter varying systems," International Journal of Innovative Computing, Information \& Control, vol. 9, no. 10, pp. 4193-4204, 2013.

[5] C. K. Ahn and P. S. K. New, "Energy-to-peak FIR filter design for systems with disturbance: a convex optimization approach," International Journal of Innovative Computing Information \& Control, vol. 9, no. 5, pp. 1987-1993, 2013.

[6] Y. S. Ledyacv and Q. J. Zhu, "Techniques for nonsmooth analysis on smooth manifolds i: local problems," Lecture Notes in Control and Information Sciences, vol. 301, pp. 283-297, 2004.

[7] Y. S. Ledyacv and Q. J. Zhu, “Techniques for nonsmooth analysis on smooth manifolds ii: deformat-ions and folws," Lecture Notes in Control and Information Sciences, vol. 301, pp. 299-311, 2004.

[8] D. Azagra, J. Ferrera, and F. López-Mesas, "Nonsmooth analysis and Hamilton-Jacobi equations on Riemannian manifolds," Journal of Functional Analysis, vol. 220, no. 2, pp. 304-361, 2005.

[9] D. Azagra and J. Ferrera, "Proximal calculus on Riemannian manifolds," Mediterranean Journal of Mathematics, vol. 2, no. 4, pp. 437-450, 2005.

[10] S. Y. Liu, S. H. Zhu, and G. Xiao, "Fritz John necessary optimality condition on Riemannian manifolds," Journal of Liaoning Normal University, vol. 30, pp. 268-272, 2007.

[11] G. Xiao and S. Y. Liu, "Necessary optimality conditions of nondifferentiable multiobjective programming on Riemannian manifolds," Journal of Jilin University Science Edition, vol. 46, no. 2, pp. 209-213, 2008.

[12] J.-H. Wang, J.-C. Yao, and C. Li, "Gauss-Newton method for convex composite optimizations on Riemannian manifolds," Journal of Global Optimization, vol. 53, no. 1, pp. 5-28, 2012.

[13] G. C. Bento, O. P. Ferreira, and P. R. Oliveira, "Unconstrained steepest descent method for multicriteria optimization on riemannian manifolds," Science Citation Index, vol. 154, pp. 88107, 2012.

[14] S. Lang, Analysis II, Addison-Wesley, 1969.

[15] W. W. Fu, Optimality Conditions on Riemannian Manifold of Nonsmooth Optimization, Liaoning Technical University, 2009. 


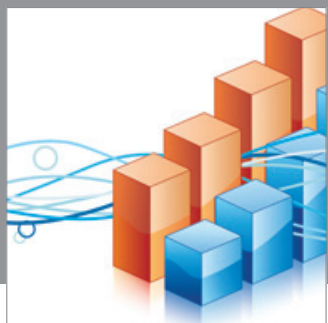

Advances in

Operations Research

mansans

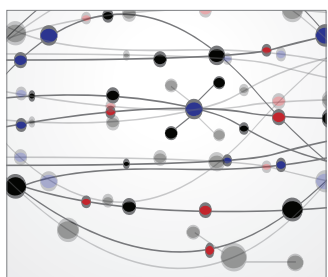

The Scientific World Journal
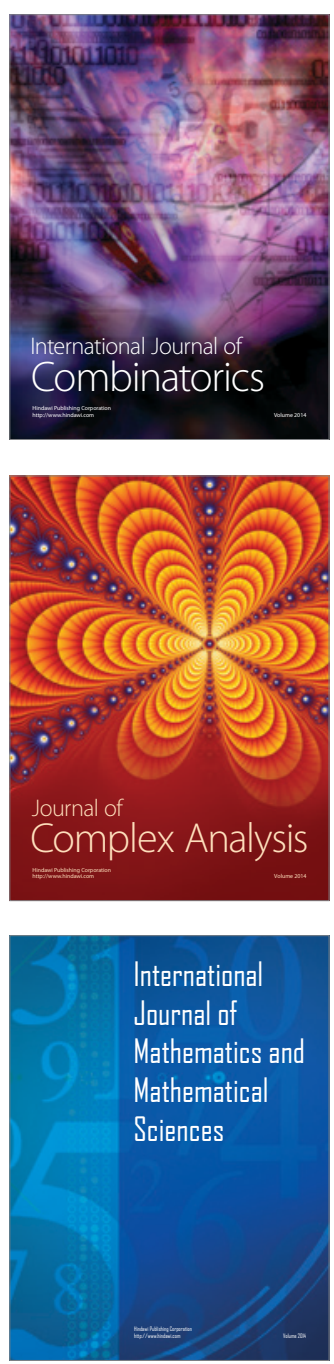
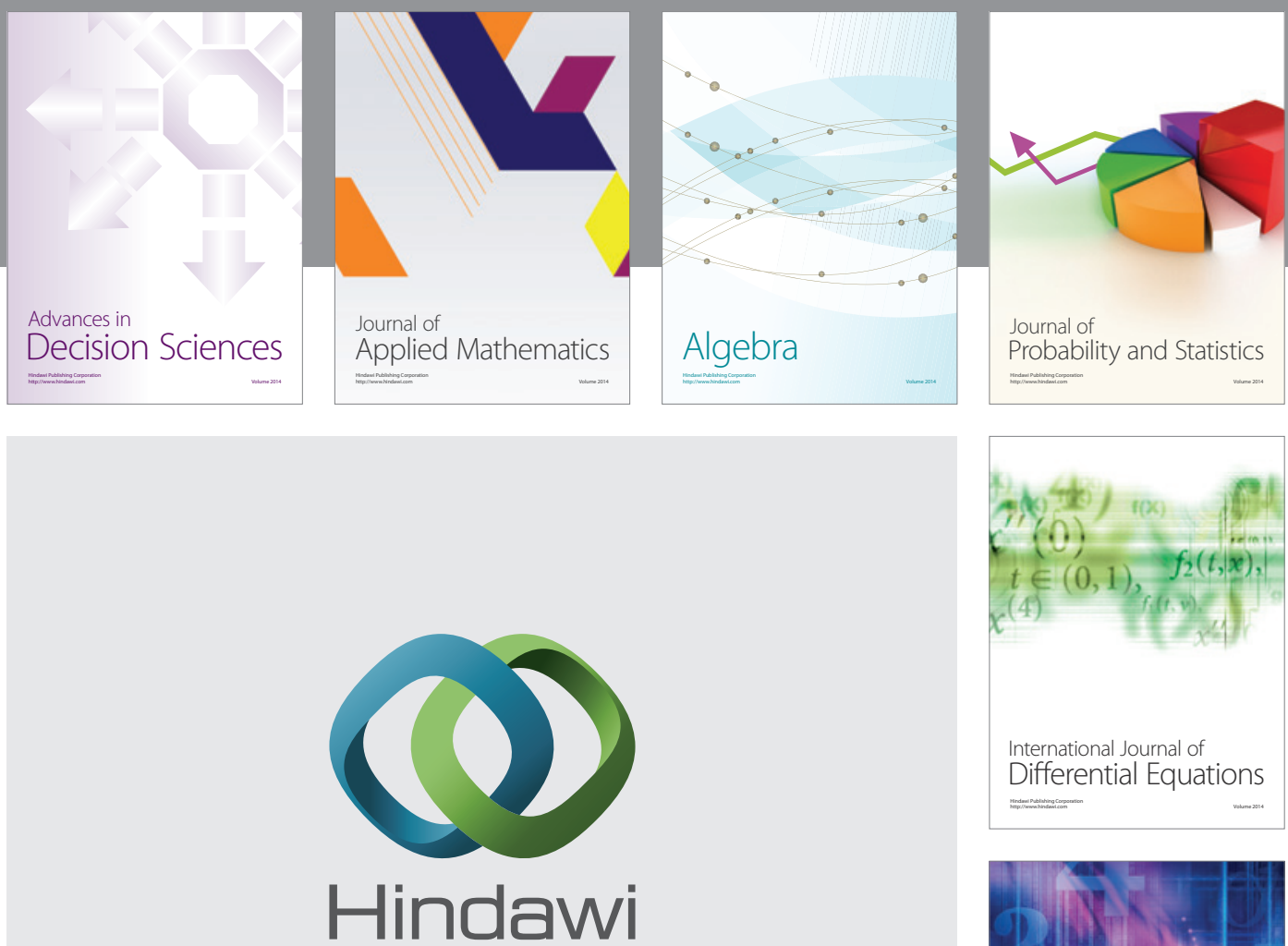

Submit your manuscripts at http://www.hindawi.com
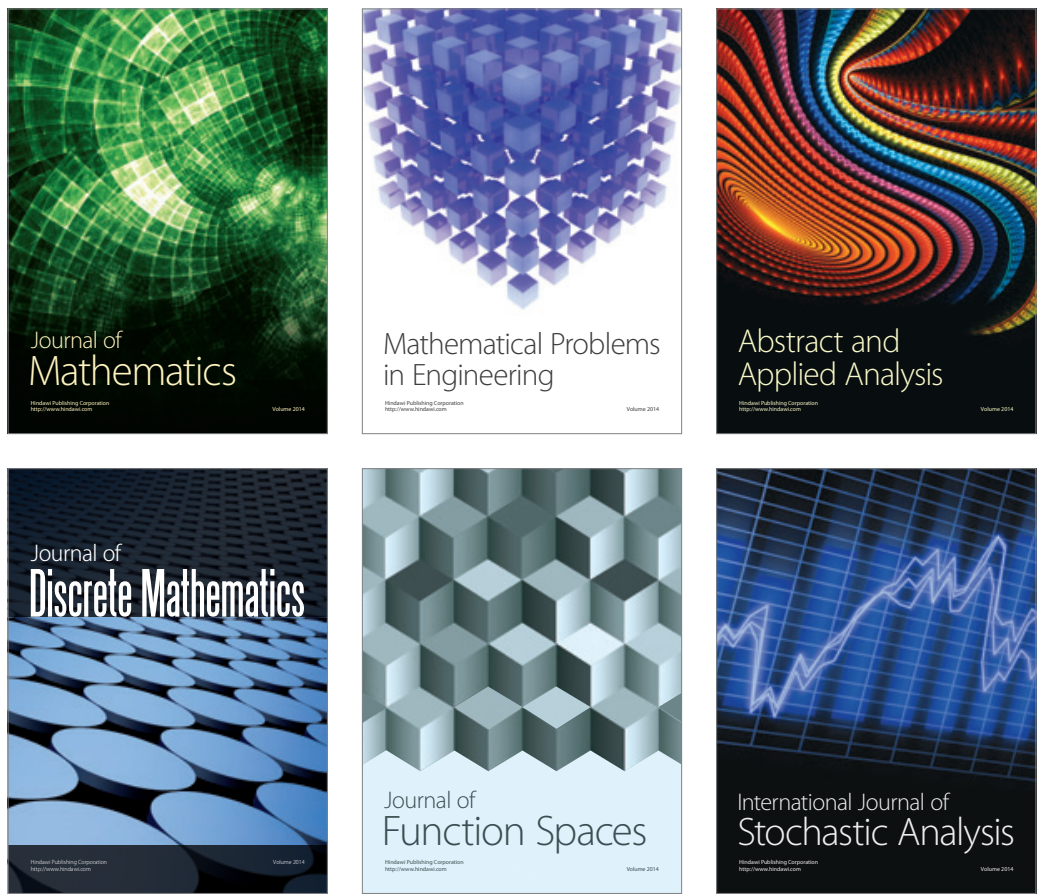

Journal of

Function Spaces

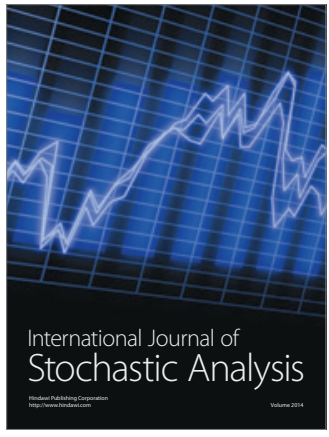

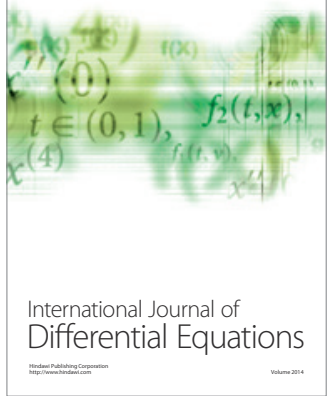
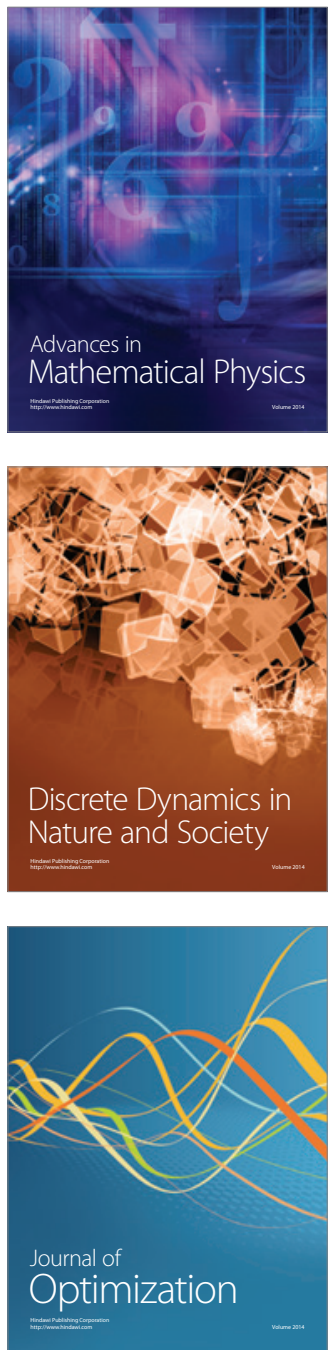